\title{
Salutogenesis in Challenging Social Circumstances and Environments
}

\author{
Bengt Lindström
}

\section{Introduction}

Part VII of the Handbook is new to the 2nd Edition, addressing the utility of a salutogenic approach in coping with extreme and challenging environments. This is obviously in line with Aaron Antonovsky's original study of menopausal women who, despite having faced extreme stress earlier in life, managed to do well at the time of his study (Antonovsky et al., 1971). This came as a complete surprise to Antonovsky and aroused his curiosity, driving him to address the question, how could these women possibly do well after their experience of extreme adversity? His answer, as we all know, was a strong sense of coherence. His quest, as we are keenly aware, led to the salutogenic model of health. In the chapters of Part VII, we are on a path following Antonovsky's footsteps, seeking constructive solutions in seemingly impossible situations. The settings included in the chapters are heterogeneous and stem from diverse geographic locations.

Chapter 46 by Bakibinga and Matanda examines the potential of a salutogenic orientation to childcare in poor urban African settings. There is an as of yet limited but growing body of evidence on the application of the salutogenic model in research among children who experience adversity in Sub-Saharan Africa (SSA). For example, a study in Uganda explored challenges faced by orphaned children in group homes, their coping strategies, as well as their ability to thrive.

Thriving was supported by generalized resistance resources (GRRs) that promoted coping with challenges, especially attention from caregivers. The authors discuss the need for more childcare research in Africa, using a salutogenic lens. As most salutogenic research with children has been conducted on other continents, the authors call for collaborations between African scholars and salutogenesis researchers in other parts of the world.

B. Lindström $(\bowtie)$

NTNU Center for Health Promotion Research, Norwegian University of Science and Technology, Trondheim, Norway

e-mail: bengtblind@ hotmail.com
Chapter 47 by Daniel and Ottemöller gives a brief overview of research on salutogenesis and migration, including both forced and voluntary migration. Salutogenesis has been used to frame labor migration and how people respond and adapt to new cultural contexts.

The focus is mainly on forced migration, i.e., the case of refugees. The authors consider research framed by the Salutogenic Model of Health (Model) and research with refugees that uses the broader "salutogenic" approach. They conclude by discussing how salutogenesis adds insight-but may also induce distraction-in the study of refugee migration.

Chapter 48 by Golembiewski and Zeisel addresses salutogenic approaches in dementia care support, using a residentcentered model of care. Securing patients' sense of coherence in care settings requires shifting the locus of decision-making power from only staff, to include residents. In this approach, patients manage more tasks themselves, they get what they need but also what they want, and they engage meaningfully with others and with life in general. The authors explain that implementing salutogenic models of dementia care is not a simple task. It involves reimagining approaches to interpersonal communication, the thoughtful development of meaningful and enjoyable activities, and creative inclusion and engagement of friends and family. Supportive design of facilities includes spaces that provide choice, opportunities for social interaction, and memory-triggering cues that inform patients about where they are, who they are, who other people are-in sum, environments that remind them that they are safe and happy. The authors contend that replacing old-fashioned approaches to care with life-affirming environments is richly rewarding. They explain that success in making this switch requires professionals to pivot away from models that see dementia primarily as a disease to be cured, toward seeing living with dementia in terms of maximum health and well-being. They conclude that salutogenesis is a useful theory to guide this transition.

Chapter 49 by Généreux, Roy, O’Sullivan, and Maltais has its starting point in 2013, when a train carrying crude oil 
derailed in Lac-Mégantic, Quebec, Canada. Research on the aftermath of this tragedy indicates that the adverse psychosocial impacts resulting from the rail tragedy decreased over time. The authors explain that although the tragedy certainly has left its mark, the local community is gradually adapting to its new reality. The asset-based approach to recovery that has been encouraged seems to have contributed to the "new reality," emphasizing the importance of social capital to activate individual and community resilience in post-disaster contexts. The authors identify and discuss success factors supporting the recovery of citizens and the social reconstruction of the community, and they document the positive development of the psychosocial situation in Lac-Mégantic, commenting also on the importance of developing a shared understanding of risks and working together in finding solutions.

The authors conclude by discussing the importance of long-term initiatives to promote understanding, preventing, and reducing psychosocial risks in the months and years following a disaster, and the need to move from disaster management to risk management logic in response to disasters.

Chapter 50 by Antonovsky addresses salutogenesis and the mental health of first responders (FRs). Research has observed FRs to be prone to psychological distress and psychopathology resulting from their repeated exposure to potentially traumatic events. Most of the literature is focused on post-event treatment. The author discusses a mental fitness model that includes salutogenically-oriented psychoeducation and other activities, to enhance mental fitness among FRs and build their psychological strengths as they face adversities on their job.

In closing, the author recommends that besides psychopathology-oriented programs intended for providing mental first aid to FRs and for the communities who experience potentially traumatic events, intervention also should include salutogenically-based mental preparation programs. These should emphasize the strengths and resources that could help FRs arrive at scenes of disaster equipped with salutogenic resources, at the strategic as well as tactical levels.

Chapter 51 by Woodall, de Viggiani, and South concludes Part VII, with a focus on salutogenesis in prisons. This chap- ter presents and debates how prison health rhetoric, policy, and practice are influenced by a pathogenic view of prisoner "health." The authors comment that there is growing recognition of a salutogenic approach to prison health policy and practice, to help tackle the root causes of health, criminality, and inequality. The chapter emphasizes that while the health of prisoners is influenced by material and social factors beyond their control, a salutogenic approach offers an alternative way of delivering public health and health promotion in prisons. The chapter concludes noting that the application of salutogenesis in prisons is in its infancy. They call for research, policy, and practice framed by a salutogenic orientation, leading to sustained and effective measures to improve the health of people in criminal justice settings, and reducing health inequalities in prisons.

\section{Conclusions}

Binding all these chapters together are their discussions of how the salutogenic approach can improve coping in times of severe distress, and the advantage of having salutogenic theory as a backbone for design and implementation of prevention and preparation interventions. In their conclusions, the authors illuminate factors, processes, and conditions leading to successful salutogenic management.

Although the cases presented in Part VII represent quite different realities, this variation helps to demonstrate different salutogenic applications in diverse, challenging social circumstances and environments. The advantage of this diversity is that it enriches the reader's spectrum of ideas about the application of salutogenesis when people, groups, and whole communities experience particularly adverse life events.

\section{Reference}

Antonovsky, A., Maoz, B., Dowty, N., \& Wijsenbeek, H. (1971). Twenty-five years later: A limited study of the sequelae of the concentration camp experience. Social Psychiatry, 6(4), 186-193.

Open Access This chapter is licensed under the terms of the Creative Commons Attribution 4.0 International License (http://creativecommons. org/licenses/by/4.0/), which permits use, sharing, adaptation, distribution and reproduction in any medium or format, as long as you give appropriate credit to the original author(s) and the source, provide a link to the Creative Commons license and indicate if changes were made.

The images or other third party material in this chapter are included in the chapter's Creative Commons license, unless indicated otherwise in a credit line to the material. If material is not included in the chapter's Creative Commons license and your intended use is not permitted by statutory regulation or exceeds the permitted use, you will need to obtain permission directly from the copyright holder. 